\title{
Inhibitory Activities on Mammalian Central Nervous System Receptors and Computational Studies of Three Sesquiterpene Lactones from Coriaria ruscifolia subsp. ruscifolia
}

\author{
Claudia Pérez, ${ }^{*}, a, b$ José Becerra, ${ }^{a, b}$ Paula Manríquez-Navarro, ${ }^{a}$ Luis Gerardo Aguayo, ${ }^{b, c}$ \\ Jorge Fuentealba, ${ }^{b, c}$ José Leonardo GuZmán, ${ }^{b, c}$ Pedro Joseph-NATHan, ${ }^{d}$ Verónica JimÉnez, ${ }^{e}$ \\ Marcelo Andrés MuÑoz, ${ }^{f}$ and Mario Silva ${ }^{a, b}$ \\ ${ }^{a}$ Laboratorio de Química de Productos Naturales, Departamento de Botánica, Facultad de Ciencias Naturales y \\ Oceanográficas, Universidad de Concepción; ${ }^{b}$ Centro de Investigación en Ecosistemas de la Patagonia (CIEP), \\ Universidad de Concepción; ' Laboratorio de Neurofisiología, Departamento de Fisiología, Facultad de Ciencias \\ Biológicas, Universidad de Concepción; ${ }^{e}$ Laboratorio de Química Teórica y Computacional, Departamento de Química, \\ Facultad de Química, Universidad de Concepción; Casilla 160-C, Concepción, Chile: ${ }^{d}$ Departamento de Química, Centro \\ de Investigación y de Estudios Avanzados del Instituto Politécnico Nacional; Apartado 14-740, México, D. F., 07000 \\ México: and ${ }^{f}$ Instituto de Química, Facultad de Ciencias, Universidad Austral de Chile; Valdivia, Chile.
}

Received June 15, 2010; accepted November 28, 2010; published online December 1, 2010

The electrophysiological characterization of sesquiterpene lactones from Coriaria ruscifolia subsp. ruscifolia has been tested on hippocampal neurons. The results for glycinergic rat hippocampal transmission and native $\gamma$ aminobutyric acid (GABA)ergic transmission on neurons (13DIV) are remarkably different for tutin, coriamyrtin, and dihydrotutin, being tutin the most potent inhibitor and dihydrotutin the least potent one. To understand the applied mechanism of action, we discuss the structural and electronic requirements for inhibitory activity by these sesquiterpene lactones when modulating receptors of the central nervous system. The structural and electrostatic properties of these compounds were compared to those of more active metabolites like picrotoxins. The minimal energy level of these structures was calculated and then optimized at the ab initio B3LYP/DGDZVP level of theory using Gaussian $03 \mathrm{~W}$ software. This allowed calculation of the corresponding vibrational circular dichroism spectrum of coriamyrtin which rendered the molecular absolute configuration after comparison with an experimental spectrum. These results are consistent with those from studies of other models that provide the basis for the activity on the presence of the lactone at carbons 3 and 5 , the presence of the hydroxyl group at position 6 , and the different electronic distributions observed in tutin and coriamyrtin. The latter has an isopropenyl moiety at carbon 4 in contrast to the dihydrotutin isopropyl group at the same position, which could explain the difference in activity between dihydrotutin and tutin or coriamyrtin. The presence of the hydroxyl group at carbon 2 is not decisive since this functionality is present in tutin, the most active compound, and in dihydrotutin, the less active one.

Key words tutin; coriamyrtin; dihydrotutin; picrotoxin compound; vibrational circular dichroism

The picrotoxin-like sesquiterpene lactones found in Anamirta paniculata and $A$. cocculus have generated longstanding theoretical interest, with early studies done in the 1960s and 1970s, to understand their mode of anticonvulsive action as potent transmission inhibitors in the central nervous system. The presence of an unusual carbon skeleton in the picrotoxin group of sesquiterpenes and in structurally related compounds generated crystallographic, biogenetic and conformational studies. Since then subsequent works have contributed to the study of many diseases associated to the central nervous system.

The parameters used in the first structure-activity study of picrotoxins were media convulsive dose $\left(\mathrm{CD}_{50}\right)$ and $\mathrm{LD}_{50}$. The active compounds posses a lactone ring joining the $\mathrm{C}-3$ and $\mathrm{C}-5$ atoms (see 1), a tertiary hydroxyl group at C-6, and an isopropenyl group at C-4 (Chart 1). The study allowed selection of the active compounds and determined that the existence of the hydroxyl group, the lactone ring and the axial isopropenyl group played a determinant role in the activity on the central nervous system, although picrotoxin activity was not tested at the presynaptic inhibitory synapse level of the central nervous system. ${ }^{1)}$

The results of the assessment of picrotoxinin and related compounds activity, specifically picrotin, tutin (1), and cori- amyrtin (2) on frog spinal cords were published twenty years later. The conclusions suggested that the presence of a hydroxyl group at C-6 could have a significant affinity for a $\gamma$ aminobutyric acid (GABA) effector chloride channel, an antagonist to the effects observed on $\gamma$-aminobutyric acid but not on alanine or taurine. These experiments were tested on primary afferent terminals and for the antagonism of presynaptic inhibition. ${ }^{2}$

Other sesquiterpene lactones have appeared successively. Data obtained using nuclear magnetic resonance spectroscopy and chemical evidence contribute to the available background knowledge regarding the use of aqueous ex-

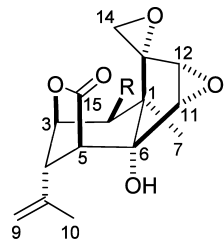

$1 \mathrm{R}=\mathrm{OH}$

$2 \mathrm{R}=\mathrm{H}$

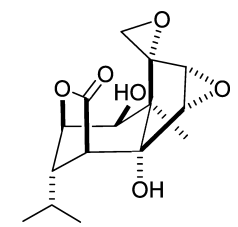

3
Chart 1. Structures of Sesquiterpene Lactones Used in This Study: Tutin (1), Coriamyrtin (2), and Dihydrotutin (3) 
tracts, amongst others of Coriaria japonica in some parts of China for the treatment of catatonia for which $\mathbf{1}$ and $\mathbf{2}$ appear to be the most active components of the extract.

Coriaria ruscifolia subsp. ruscifolia L. (Coriariaceae) is a plant native to the American Continent, distributed from Mexico through Central America and to the southern extremes of Chile, ${ }^{3)}$ where it is known as "deu," "huique," and more frequently "matarratones" (literally: mouse-killer).

The toxic effects of ingesting Coriaria ruscifolia and other species of this genus have an epileptogenic effect, ${ }^{4)}$ indirectly suggesting that the state characterized by hyperexcitability is an anti-glycinergic and/or anti-GABAergic effect triggered by the sesquiterpene lactones. Moreover, picrotoxin is a known antagonist of GABA- $R$ in all its protein isoforms and $R$-Gly in the homomeric form. ${ }^{5}$

The molecules inducing the described convulsive effects could be any of the sesquiterpene lactones present in the plant, like tutin (1), coriamyrtin (2) and/or dihydrotutin (3), although pharmacological studies have mainly been directed to the use of $\mathbf{1}$ and $\mathbf{2}$. The three molecules show small structural differences (see Chart 1) which suffice to allow 1 to modulate the R-Gly receptor, something $\mathbf{3}$ is unable to do.

In the last decade vibrational circular dichroism spectroscopy $^{6}$ (VCD) has arisen as an excellent technique to determine the absolute configuration of a given molecule or to establish the stereochemistry of one or a few chiral centers of a molecule. This also implies to ascertain the conformational preferences of this molecule since a comparison of calculated and experimental spectra is mandatory. When an investigation is set out to determine the absolute configuration, it must first establish the conformational distribution of a particular enantiomer of the molecule, usually either one, before any stereochemical conclusions can be drawn. Likewise when the absolute configuration of the studied molecule is known, theoretical and experimental VCD spectra can also be compared to ascertain or confirm the conformational preferences of a molecule, which can be of great help in the effort of understanding their pharmacological behavior.

\section{Results}

Phytochemical Analysis The sesquiterpene lactones tutin (1), coriamyrtin (2), and dihydrotutin (3) were isolated from a chloroform extract of the aerial parts of Coriaria ruscifolia subsp. ruscifolia collected in southern Chile $\left(41^{\circ} 05^{\prime} \mathrm{S}\right.$, $72^{\circ} 23^{\prime} \mathrm{W}$; Puerto Montt) and central Chile $\left(37^{\circ} 01^{\prime} \mathrm{S}\right.$, $73^{\circ} 02^{\prime} \mathrm{W}$; El Patagual, Concepción). ${ }^{7)}$ The isolation of compounds 1 and 2 has been reported from C. ruscifolia. ${ }^{8)}$ In our study compounds $\mathbf{1}$ and $\mathbf{2}$ are the main constituents in the plant, whereas dihydrotutin (3) is found in small quantities and was recently found in a collection from El Patagual.

Compound identity was established by ${ }^{1} \mathrm{H}$ - and ${ }^{13} \mathrm{C}-\mathrm{NMR}$ comparison with published values. ${ }^{9-11)}$

Theoretical Calculations. Frontier Orbital Comparison between Coriamyrtin, Tutin, and Dihydrotutin With the aim of gaining insight into the origin of the activity trends found for $\mathbf{1}-\mathbf{3}$ we carried out an electronic structure analysis by means of ab initio calculations. Since biological activity is strongly related to the solution state conformations of the active molecule and its ability to interact with the biological receptor, the most stable conformation of each molecule was obtained using the Monte Carlo-molecular mechanics
(MMFF) conformational search followed by geometry optimizations and vibrational calculations at the B3LYP/ DGDZVP of theory. Three-dimensional plots of highest occupied molecular orbital (HOMO) and lowest unoccupied molecular orbital (LUMO) for the most stable conformation of $\mathbf{1}-\mathbf{3}$ were mapped using the wave function at the same level of theory, as shown on Fig. 1.

VCD Absolute Configuration and Conformational Behavior of Coriamyrtin Table 1 shows the molecular mechanic energies and abundances derived from the Monte Carlo-MMFF conformational search, as well as the corresponding energy optimization and conformational distribution for coriamyrtin (2) which showed four low energy conformations that were ruled mainly by the isopropenyl and hydroxyl group mobility as shown in Fig. 2. Two orientations were observed for the isopropenyl moiety, with the methyl group oriented towards or against the carbonyl side of the five-membered lactone (less and more energetic conformations, respectively), and two for the hydroxyl group, with the
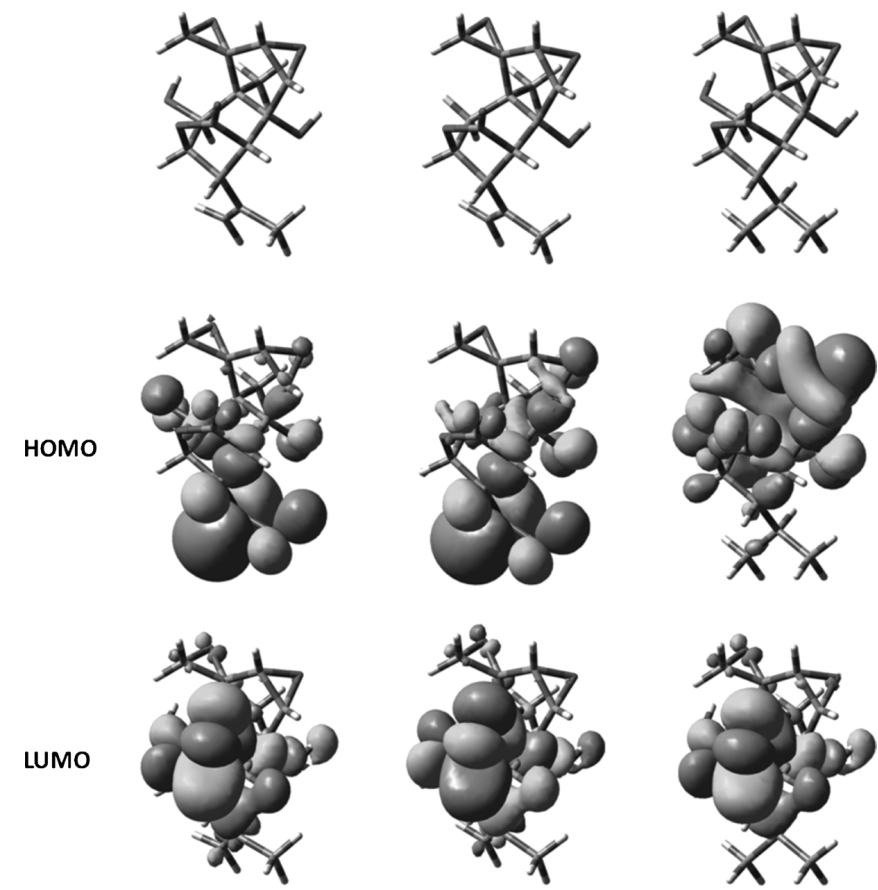

1

2

3

Fig. 1. The Most Stable Conformation (Top) and 3D Plots of Their Frontier Orbitals (HOMO Middle and LUMO Bottom) of Compounds 1 (Left), 2 (Center), and $\mathbf{3}$ (Right)

Table 1. Calculated Relative Energies $(\mathrm{kcal} / \mathrm{mol})$ and Relative Free Energies and Abundances (\%) of the Three Most Stable Conformers of 2 Using Monte Carlo Search and Geometry Optimization Calculations at the MMFF and B3LYP/DGDZVP Levels of Theory

\begin{tabular}{ccrcr}
\hline \hline Conf. & $\mathrm{E}_{\mathrm{MMFF}}{ }^{a}$ & $\%_{\mathrm{MMFF}}$ & $\Delta \mathrm{G}_{\mathrm{OPT}}{ }^{a)}$ & $\%_{\mathrm{OPT}}{ }^{b)}$ \\
\hline 2a & 0.00 & 74.91 & 0.00 & 93.85 \\
2b & 0.65 & 25.04 & 1.77 & 4.75 \\
2c & 4.50 & 0.04 & 2.54 & 1.30 \\
2d & 5.47 & 0.01 & 4.04 & 0.10
\end{tabular}

Conformers are ordered according to their relative abundance. a) Relative to the lowest energy conformer in the molecular mechanics force field ( $\mathrm{E}_{\mathrm{MMFF}} \mathbf{2 a}=61.54$ $\mathrm{kcal} / \mathrm{mol})$ and DFT $\left(\Delta G_{\text {OPT }} \mathbf{2 a}=-601337.91 \mathrm{kcal} / \mathrm{mol}\right)$ levels of theory. $b$ ) Calculated using the optimized free energies of the relevant conformers. 


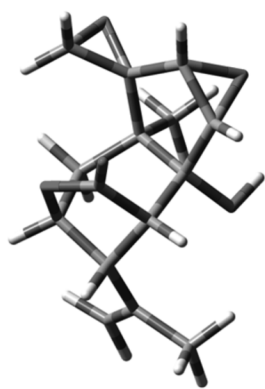

2a

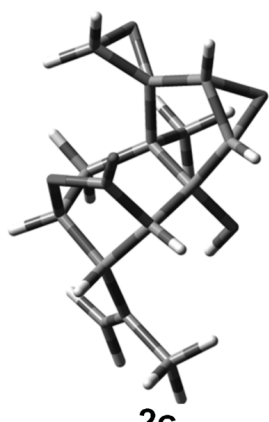

2c

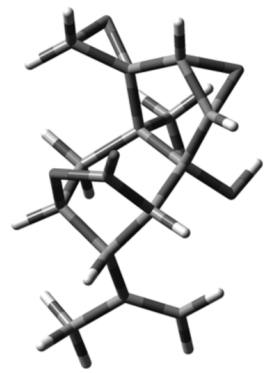

$2 b$

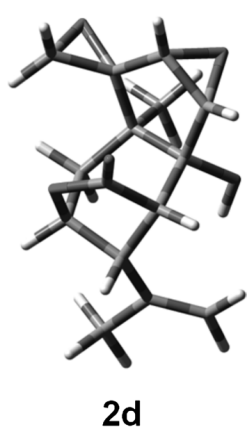

2d
Fig. 2. The Four Most Stable Conformations of Coriamyrtin (2)

hydrogen atom oriented towards the closest oxirane oxygen and under the fused cyclohexane ring (less and more energetic, respectively). Of these four, the two most stable conformations accounted for $98.6 \%$ of the entire conformational distribution, rendering unnecessary further consideration of the remaining two conformations since they would have very little impact on the molecular properties to be calculated. The two more stable conformations shared the same orientation of the hydroxyl group but differed in their disposition of the isopropenyl substituent.

For a better comparison with the experimental vibrational spectrum, a single weighted theoretical VCD spectrum was plotted from the individual spectra of each conformation using the relative abundances obtained from calculated free energies (Fig. 3).

Electrophysiological Characterization on Hippocampal Neurons To characterize the activity profile of these three molecules, we used an electrophysiological approach that allowed to determine the inhibitory potency of $\mathbf{1} \mathbf{- 3}$. First, we tested the activity of $\mathbf{1}$ and $\mathbf{2}$ on glycinergic rat hippocampal transmission using neurons with $13 \mathrm{~d}$ in vitro (DIV). We coapplied $1(50 \mu \mathrm{M})$ with the physiological agonist glycine (30 $\mu \mathrm{M})$, whereby tutin blocked $30 \pm 9 \%(n=5)$ of the control current, (glycine $30 \mu \mathrm{M}$ ). In parallel, 2 was applied using a similar protocol and induce a current inhibition about $20 \pm 6 \%(n=5)$ of the control current, both molecules do not shown significative differences in the inhibitory potency. The concentration-response curve failed to show any difference between the inhibitory effects of $\mathbf{1}$ and $\mathbf{2}$, eliciting maximal blockades of $40 \pm 10 \%$ and $39 \pm 4 \%$, respectively $(n=6)$. On the other hand, 3 showed a non significant lowest inhibitory potency, the maximal inhibitory effect of glycinergic current of $16 \pm 7 \%$. Consequently it seems the isopropyl group of 3 don't exerts an important change on the ligand receptor on glycine receptor.

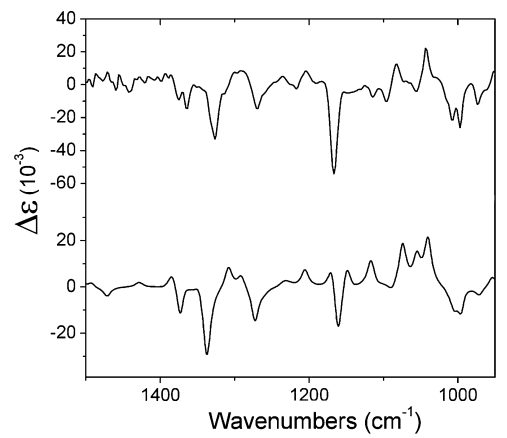

Fig. 3. Comparison of Experimental (Top) and Calculated (Bottom) VCD Spectra of Coriamyrtin (2)
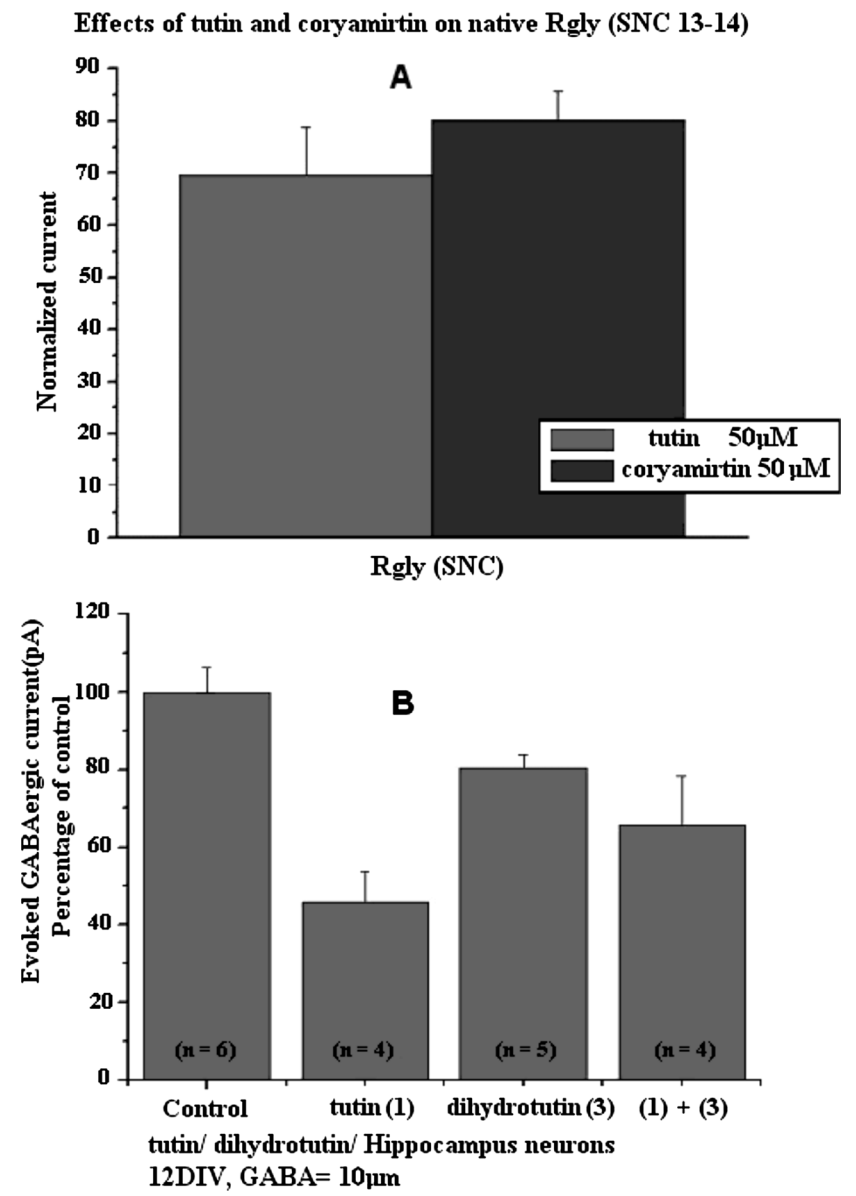

Fig. 4. Electrophysiological Characterization

(A) Coriamyrtin $(50 \mu \mathrm{M})$ not showing significant differences compared with tutin $(50 \mu \mathrm{M})$ to block the current induced by glycine $(30 \mu \mathrm{M})$ tested on spinal cord neurons (SCN, $n=5)$. (B) The effects of dihydrotutin $(50 \mu \mathrm{M})$ on GABAergic neurons compared with tutin $(50 \mu \mathrm{M})$; note that in this model the inhibitory effect elicited for dihydrotutin was smaller than that for tutin.

We also tested the inhibitory effects of these sesquiterpene lactones on native GABAergic transmission on neurons (12-14 DIV). According with the previous results, compound 3 showed a lower inhibitory potency than 1, eliciting only $20 \pm 5 \%$ blockage, while 1 induced $55 \pm 7 \%$ blockage $(n>4)$. These results suggested that the GABA receptor could be a more sensitive receptor to the inhibitory actions of molecules under study and the antagonist binding site fit better with the sesquiterpenes structures, inducing a major interference into the pore permeability. Therefore, interactions 
between key amino acids on the pore-channel must be crosslinked with analyses of the chemical structure to determine the importance of different electronic densities in the isoprenylated regions of compounds $\mathbf{1}$ and $\mathbf{2}$ to the potency or binding efficiency of the molecules for glycine or GABA (see Fig. 4).

\section{Discussion}

In the present study of Coriraria ruscifolia subsp. ruscifolia, three picrotoxin-type compounds were isolated from which tutin (1) and coriamyrtin (2) had been previously reported, ${ }^{8)}$ while dihydrotutin (3) was not observed in the specimens from Puerto Montt, but appeared in the collection from Patagual.

Okuda and Yoshida ${ }^{12,13)}$ studied the structure and absolute configuration of $\mathbf{2}$ in the early 1960s which was confirmed by total synthesis in $1984 .^{14)}$

This is the first evaluation of the direct modulation of $\mathbf{1}-\mathbf{3}$ on GlyR. The $\mathrm{IC}_{50}$ value of $\mathbf{1}$ owns to its correlation with the glycinergic receptor subtype (homomeric or heteromeric). This study showed that $\mathbf{1}$ is the most potent of the three compounds and that $\mathbf{3}$ did not produce any significant modulation on the GlyRs. These studies about the interaction of the modulation of 1 on $R$-Gly and $R-\mathrm{GABA}_{\mathrm{A}}$ receptors are promising because the results suggest that the toxic effect of the plant is due to the presence of $\mathbf{1}$.

With the objective to rationalize the intermolecular interaction that governs the activity of $\mathbf{1}-\mathbf{3}$, we analyzed the frontier molecular orbital of these compounds under the assumption that frontier orbitals must be involved in the interaction between these species and the appropriate molecular orbitals of a biological receptor. Nevertheless in order to do so, the conformational preferences of each molecule need to be asserted, and at some level confirmed experimentally. This was achieved through Monte Carlo conformational calculations from which some interesting conclusions can be drawn. First it is interesting to note that the ester linkage and epoxide rings restricted the conformational freedom of the sixand five-member rings in $\mathbf{1}-\mathbf{3}$. Therefore, one should expect only a small degree of conformational distortion when comparing solid state structures with the conformations that these species adopt in solutions or in biological media. As a consequence of this high rigidity, the bioactive conformation of these molecules were expected to be quite similar to their solid state structures and also to the optimized geometries reported herein. This was confirmed since the calculated conformations for $\mathbf{1}$ and $\mathbf{2}$ compared quite well with reported ${ }^{15}$ ) $\mathrm{X}$-ray crystal structures as evidenced by the selected dihedral angles given in Table 2 . In the case of $\mathbf{3}$ a crystal structure remains undetermined but the calculated conformation shows many of the conformational characteristics of $\mathbf{1}$ and $\mathbf{2}$, strongly suggesting that $\mathbf{3}$ is also well represented by its optimized geometry, as shown in Fig. 2.

The preferred orientation of the hydroxyl group, which formed a weak intramolecular hydrogen bond that interacted with the oxygen in the vicinal oxirane, has been suggested before by means of molecular mechanics and semiempirical AM1 calculations. ${ }^{15)}$ The same calculations also predicted the two low energy dispositions of the isopropenyl moiety and their relative order of stability. Nevertheless, none of these results were compared against the solution state data to
Table 2. Comparison of Calculated (in the Gas Phase) and Measured (Solid State Single Crystal X-Ray Diffraction) Dihedral Angles for Compounds $\mathbf{1}$ and $\mathbf{2}$

\begin{tabular}{|c|c|c|c|c|}
\hline \multirow{2}{*}{ Diedral angle } & \multicolumn{2}{|c|}{1} & \multicolumn{2}{|c|}{2} \\
\hline & X-ray ${ }^{a}$ & DFT & X-ray ${ }^{a}$ & DFT \\
\hline $\mathrm{C} 1-\mathrm{C} 2-\mathrm{C} 3-\mathrm{C} 3 \mathrm{a}$ & -3.7 & 5.3 & 1.0 & 4.5 \\
\hline $\mathrm{C} 2-\mathrm{C} 3-\mathrm{C} 3 \mathrm{a}-\mathrm{C} 7 \mathrm{a}$ & 18.1 & 11.2 & 14.4 & 11.1 \\
\hline $\mathrm{C} 3-\mathrm{C} 3 \mathrm{a}-\mathrm{C} 7 \mathrm{a}-\mathrm{C} 1$ & -24.2 & -22.4 & -23.1 & -21.4 \\
\hline $\mathrm{C} 3 \mathrm{a}-\mathrm{C} 7 \mathrm{a}-\mathrm{C} 1-\mathrm{C} 2$ & 22.8 & 26.2 & 24.4 & 24.8 \\
\hline $\mathrm{C} 7 \mathrm{a}-\mathrm{C} 1-\mathrm{C} 2-\mathrm{C} 3$ & -12.8 & -20.3 & -16.7 & -19.0 \\
\hline $\mathrm{C} 3 \mathrm{a}-\mathrm{C} 4-\mathrm{C} 5-\mathrm{C} 6$ & -72.7 & -70.8 & -71.3 & -71.4 \\
\hline $\mathrm{C} 4-\mathrm{C} 5-\mathrm{C} 6-\mathrm{C} 7$ & 70.2 & 71.8 & 68.1 & 70.6 \\
\hline $\mathrm{C} 5-\mathrm{C} 6-\mathrm{C} 7-\mathrm{C} 7 \mathrm{a}$ & -55.7 & -58.6 & -52.2 & -54.0 \\
\hline C6-C7-C7a-C3a & 33.9 & 32.7 & 28.8 & 27.5 \\
\hline $\mathrm{C} 7-\mathrm{C} 7 \mathrm{a}-\mathrm{C} 3 \mathrm{a}-\mathrm{C} 4$ & -35.6 & -29.8 & -30.0 & -26.6 \\
\hline $\mathrm{C} 7 \mathrm{a}-\mathrm{C} 3 \mathrm{a}-\mathrm{C} 4-\mathrm{C} 5$ & 59.6 & 53.6 & 55.9 & 53.1 \\
\hline C4-C5-C6-O14 & -46.3 & -46.8 & -48.3 & -47.1 \\
\hline C5-C6-O14-C12 & 30.1 & 30.4 & 33.6 & 31.2 \\
\hline C6-O14-C12-C4 & 0.0 & 0.0 & -3.9 & -1.1 \\
\hline $\mathrm{O} 14-\mathrm{C} 12-\mathrm{C} 4-\mathrm{C} 5$ & -29.3 & -29.8 & -26.2 & -28.9 \\
\hline C12-C4-C5-C6 & 44.6 & 45.1 & 44.2 & 44.9 \\
\hline C5-C4-C12-C13 & 152.4 & 150.5 & 153.2 & 151.3 \\
\hline C4-C5-C15-C16 & -132.7 & -139.8 & -132.8 & -138.2 \\
\hline C4-C5-C15-C17 & 54.1 & 48.2 & 56.4 & 49.7 \\
\hline C6-C5-C15-C16 & -12.4 & -17.3 & -11.5 & -16.1 \\
\hline O11-C3a-C7a-C18 & -26.1 & -27.8 & -30.8 & -26.7 \\
\hline C18-C7a-C7-O19 & -75.7 & -80.9 & & \\
\hline
\end{tabular}

a) Data taken from ref. 15 .

ascertain the preferences of $\mathbf{2}$ in this medium. Therefore in order to do this confirmation, the theoretical and experimental VCD spectra of 2 were compared (Fig. 3) revealing both traces share the same features, thus confirming the calculated conformations to be those preponderant in solution.

As mentioned earlier, the absolute configuration used in the calculations was the one proposed by Okuda and Yoshida, ${ }^{12,13)}$ and as can be concluded by later comparison, it corresponded to the proposed one. Furthermore, the absolute configurations of tutin (1) and dihydrotutin (3) were the same as that of 2, as evidenced through chemical transformations.

Using these low energy conformations, the 3D contour plots of the highest-occupied and lowest-unoccupied molecular orbital (HOMO and LUMO, respectively) were obtained and are shown in Fig. 1. According to frontier molecular orbital theory, the most suitable interactions in a molecule are those that provide the largest contributions to the frontier orbitals. Therefore, the location of both HOMO and LUMO in each species should be helpful for identifying the sites able to interact with the appropriate molecular orbital of the biological receptor, explaining the trends discussed before. Accordingly, Fig. 1 shows that the HOMO in $\mathbf{1}$ and $\mathbf{2}$ is mainly located on the isopropenyl moiety whereas, for compound $\mathbf{3}$, this frontier orbital is spread along the five and six-membered rings, showing a change in the most suitable interaction sites due to the saturation of the isopropenyl moiety. On the contrary, the LUMO orbital is located in the same area in all three molecules. These findings agree with the biological activity trends observed for these compounds.

At present, receptors knowledge is quite widespread, although it is still insufficient and there is a need to characterize the pharmacological parameters and to model it in vitro and in vivo by means of physiological experiments and spe- 
cially its chemical characteristics like structure, conformation, ability to form hydrogen bridge links, electron density, and so on, that enable it to interact with a host site.

A good number of these molecules show strong activity on neural systems and possess a similar pharmacological profile, which is why we find a good number of studies on structure-activity relationships for these kinds of substances. For example, studies have been done with insect and mammal receptors and combinations of the two to establish animal models of epilepsy, converting this into a valuable pharmacological tool in the study of various physiological manifestations of the central nervous system. ${ }^{16)}$

\section{Experimental}

Chemicals The sesquiterpene lactones; tutin (1), coriamirtyn (2) and dihydrotutin (3) used in the present study were isolated from Coriraria ruscifolia subsp. ruscifolia. The methodology used was similar to that described. ${ }^{8)}$

Computational Details Conformational searches were carried out based on molecular mechanics (MMFF) as implemented in the Spartan'04 software package ${ }^{17)}$ and considering an energy cutoff of $10 \mathrm{kcal} / \mathrm{mol}$ above the global minimum. All conformations derived from the search were further optimized at the B3LYP/DGDZVP level of theory and their free energies were obtained using vibrational calculations at the same level of theory. Three-dimensional plots of HOMO and LUMO for $\mathbf{1}-\mathbf{3}$ were mapped using the B3LYP/DGDZVP wave function of the most stable conformation of each molecule. For 2, the frequencies, as well as dipole and rotational strengths were extracted from the vibrational calculations and weighed plots using the corresponding free energies were produced considering Lorentzian bands with half-widths of $6 \mathrm{~cm}^{-1}$ and an anharmonicity factor of 0.97. Ab initio calculations were carried out using the Gaussian $03 \mathrm{~W}$ software package. ${ }^{18)}$

Electrophysiological Recordings. Animal Testing and Biological Data Part of the data was extracted from our previous report. ${ }^{19)}$

Cell Culture Mouse (C57BL/J6) spinal cord neurons obtained from five to six embryos $(13-14 \mathrm{~d})$ were plated at $300000 \mathrm{cells} / \mathrm{ml}$ into $35-\mathrm{mm}$ tissue culture dishes coated with poly-L-lysine (MW $350 \mathrm{kDa}$, Sigma Chemical, St. Louis, MO, U.S.A.). The neuronal feeding medium consisted of $90 \%$ minimal essential medium (GIBCO, Grand Island, NY, U.S.A.), 5\% heat-inactivated horse serum (GIBCO), $5 \%$ fetal bovine serum (GIBCO), and a mixture of nutrient supplements. Fresh media was replaced every $3 \mathrm{~d}$. In vitro neuron experiments were performed for $14 \mathrm{~d}$.

Electrophysiological Recordings Voltage-clamp recordings were performed in a whole cell configuration and acquired with an Axon 200-B amplifier (Axon Instruments, Union City, CA, U.S.A.). Patch-clamp microelectrodes were filled with (in mM): $140 \mathrm{KCl}, 10$ BAPTA, $10 \mathrm{~N}$-(2-hydroxyethyl)piperazine- $N$ '-2-ethanesulfonic acid (HEPES) ( $\mathrm{pH} 7.4$ ), $4 \mathrm{MgCl}_{2}, 0.3$ guanosine 5 '-triphosphate (GTP), and 2 ATP-Na, 300 mOSM. The external solution c ontained (in mM): $\mathrm{NaCl}$ (150), $5 \mathrm{KCl}$ (5.4), $\mathrm{CaCl}_{2}(2.0), \mathrm{MgCl}_{2}(1.0)$, 10 HEPES ( $\mathrm{pH} 7.4$ ), and 10 glucose. The holding potential was fixed at $-60 \mathrm{mV}$; recordings were filtered at $5 \mathrm{kHz}$ with a low pass Bessel filter. Fresh solutions were prepared daily. The recordings were made by applying short ( $2 \mathrm{~s})$ pulses of glycine $(30 \mu \mathrm{M})$ every $1 \mathrm{~min}$ with a rapid perfusion system. After stabilizing the glycinergic current amplitude, 1 was co-applied and the amplitude of the current to glycine was re-evaluated. We applied the full range of tutin concentrations $(1-1000 \mu \mathrm{M})$ to each neuron in order to obtain a concentration-response curve. The decay time constant, amplitude, frequency, and number of events were analyzed with the Minianalysis program (Synaptosoft Inc., U.S.A.).

Acknowledgments This work was supported by Proyecto DIUC-Patagonia 205.111.45-1SP. (Universidad de Concepción). Patagonian Ecosystems Research Center (CIEP), Proyecto Anillo ADI-38, Proyecto INNOVA BIO BIO 05B1397L7 and CONACYT-Mexico grant no. 61122.

\section{References}

1) Jarboe C. H., Porter L. A., Buckler R. T., J. Med. Chem., 11, 729-731 (1968).

2) Kudo Y., Niwa H., Tanaka A., Yamada K., Br. J. Pharmacol., 81, 373-380 (1984).

3) Skog L., Rodhora, 74, 242-253 (1972).

4) Zhou H., Tang Y.-H., Zheng Y., Brain Res., 1092, 207-213 (2006).

5) Olsen R. W., Proc. Natl. Acad. Sci. U.S.A., 103, 6081—6082 (2006).

6) Nafie L. A., Nat. Prod. Commun., 3, 451-466 (2008).

7) Marticorena C., Rodríguez R., "Flora de Chile," 2nd ed., Universidad de Concepción, Concepción, Chile, 2003.

8) Reyes A., García-Quintana H., Romero M., Morales D., Bartulin J., Bol. Soc. Chil. Quím., 43, 87-89 (1998).

9) Aguirre-Galviz L. E., Fitoterapia, 58, 50-51 (1987).

10) Koike K., Susuki Y., Ohmoto T., Phytochemistry, 35, 701-704 (1994).

11) Perry N. B., Aiyez M., Kerr D. S., Lake R. J., Leach M. T., Phytochem. Anal., 12, 69-72 (2001).

12) Okuda T., Yoshida T., Tetrahedron Lett., 1964, 439-444 (1964).

13) Okuda T., Yoshida T., Tetrahedron Lett., 1965, 4191 - 4197 (1965).

14) Niwa H., Wakamatsu K., Hida T., Niiyama K., Kigoshi H., Yamada M., Nagase H., Suzuki M., Yamada K., J. Am. Chem. Soc., 106, 4547-4552 (1984).

15) Gulbis J. M., Mackay M. F., Wong M. G., Aust. J. Chem., 42, 18811896 (1989).

16) Ozoe Y., Akamatsu M., Higata T., Ikeda I., Mochida K., Koike K., Ohmoto T., Nikaido T., Bioorg. Med. Chem., 6, 481- 492 (1998).

17) Spartan '04, Wavefunction, Irvine, CA (2004).

18) Gaussian 03W, Gaussian Inc., Pittsburgh, PE (2003).

19) Fuentealba J., Guzman L., Manríquez-Navarro P., Pérez C., Silva M., Becerra J., Aguayo L. G., Eur. J. Pharmacol., 559, 61-64 (2007). 